\title{
On faithful representations of free products of groups
}

\section{by}

\section{S. Balcerzyk (Toruń) and Jan Mycielski (Wrocław)}

1. $O_{3}$ denotes tho group of real orthogonal matrices, with doterminant equal to 1, acting on $\chi^{3}$. 'M denotes the group of substitutions $w=(a z+b) /(c z+d)$ with real $a, b, c, d$ and $a d-b c=1$.

Our ehief result is the following $\left({ }^{1}\right)$ :

THEOREM 1. If $G_{t}(t \in T)$ is a system of groups such that each $G_{t}$ is isomorphic to a subgroup of $\mathcal{O}_{3}$ or each $G_{t}$ is isomorphic to a subgroup of $m, \overline{G_{t}}<2^{\aleph_{0}}$ for each $t \in T$ and $\bar{T} \leqslant 2^{\aleph_{0}}$ then the free product $\prod_{t \in T}^{*} G_{t}$ is isomorphic to a subgroup of $\mathrm{O}_{3}$ or $M$, respectively.

This theorem (in the case of $O_{3}$ ) was conjectured by J. do Groot ([11]). It is a finalisation of a long list of results: F. Hausdorff ([12]) has proved that the free product $Z_{2} * Z_{3}\left(Z_{n}\right.$ denotes the eyclic group of order $n \leqslant \infty)$ is isomorphic to a subgroup of $O_{3}$. Therefore the free group $\prod_{n=1}^{\infty} Z_{\infty}$ is isomorphic to a subgroup of $\mathrm{O}_{3}$ (by the elementary fact that each group of the form $G * H$ contains a subgroup isomorphic to $\prod_{n=1}^{\infty} Z_{\infty}$, except in the case when $G$ or $H$ is the unity group, or $\left.G \cong H \cong Z_{2}\right)$. W. Sierpinski ([20] Lemme 1) has proved that the free group $\prod_{t \in \mathscr{R}}^{*} Z_{\infty}$ of potency $2^{\mathrm{N}_{0}}$ is isomorphic to a subgroup of $\mathcal{O}_{3}$. All these restilts were obtained for the purpose of the s.c. paradoxical decompositions. J. de Groot ([11]) has studied the problem for himself and has given a simple proof and some improvements of the result of Sierpiriski. Th. J. Dokker ([9]) has proved that $\prod_{n<\infty}^{*} \prod_{t \in \mathscr{R}}^{*} Z_{n}$ is isomorphic to a subgroup of $O_{3}\left({ }^{2}\right)$.

(1) The main results of this paper were announced withouti prool in [1]. Theorem It was then known to us in a somewhat weaker form improved now by the applisation of a result of S. Swierczkowski (see footnote (7)). Another result announced in [1], section 3, concerning the representations of free products by perrnutation groups will not be studied here since it has been independently obtained and refined by $N$. 4 . de Bruijn ([4], [5], [6]).

(2) For other investigations related to our subject see [3], [7], [8], [18]. 
Concerning the group $M$ it is known that $Z_{2} * Z_{3}$ is isomorphic to the subgroup of substitutions in which $a, b, c, d$ are integers ( $\left.{ }^{3}\right)$.

All these results were obtained without the use of the axiom of choice. Our theorem which generalises of course all of them is obtained by a different method and applies the axiom of choice. It should be mentioned that the continuum hypothesis is not applied, due to a method developed in [2].

It seems plausible that Theorem 1. holds if $\mathrm{O}_{3}$ or $9 / \mathrm{T}$ is replaced by any simple connected Lie group $\mathcal{G}$.

In Section 2 we give some general results reducing this problem to the proof of a lemma $L(g)$ which wo have boon able to estiablish only for $\mathrm{O}_{3}$ and $m$. The proof of $L\left(\mathrm{O}_{3}\right)$ and $L(m)$ follows in soction 3 . In Section 4 we prove other results on $\mathrm{O}_{3}$ solving another problem of $\mathrm{J}$, de Groot ([11]).

2. $G$ denotes any group (the group operations are written multiplicatively and the unity is denoted by 1 ).

For every set $K \subset G$ we denote by $[K]$ the subgroup of $G$ generated by $K$.

For a system of groups $G_{t}(t \in T)\left(^{4}\right)$ we denote by $\prod_{t \in T^{*}}^{*} G_{t}$ the free product of this system, that is the group generated by the set of all ordered pairs $\langle g, t\rangle$ with $t \in T, g \in G_{t}$ determined by the relations

$$
\begin{aligned}
\langle g, t\rangle^{-1} & =\left\langle g^{-1}, t\right\rangle, \\
\left\langle g_{1}, t\right\rangle\left\langle g_{2}, t\right\rangle & =\left\langle g_{1} g_{2}, t\right\rangle, \\
\left\langle 1, t_{1}\right\rangle & =\left\langle 1, t_{2}\right\rangle .
\end{aligned}
$$

If we have a system of homomorphisms $h_{t}: G_{t} \rightarrow G$, then the natural homomorphism $\left.h:]_{t \in T}\right]_{T}^{*} G_{t} \rightarrow\left[\bigcup_{t \in T} h_{t}\left(G_{t}\right)\right]$ is determined by $h(\langle g, t\rangle)=h_{t}(g)$. In particular, if $G_{t} \subset G$ and $x_{t} \in G(t \in T)$ then the natural homomorphism $\prod_{i \in T}^{*} G_{t} \rightarrow\left[\bigcup_{t \in T} x_{t} G_{t} x_{t}^{-1}\right]$ is determined by $\langle g, t\rangle \rightarrow x_{t} g x_{t}^{-1}$.

Let us consider the following proposition.

$L(G)$. For any $a_{1}, \ldots, a_{m} \in G \backslash(1)$ and any integers $k_{1}, \ldots, k_{m}$ different from $0(m=1,2, \ldots)$ the function of the variable $x \in G$

$$
a_{1} x^{k_{1}} a_{2} x^{k_{2}} \ldots a_{m} x^{k_{m}}
$$

is not identically equal to 1.

$g$ denotes any connected Lie group.

$\left({ }^{3}\right)$ A simple proof is given by K. A. Hirsch [13]. For other resultis and references see [8], [10], [11], [13], [19].

(4) We do not suppose that $t_{1} \neq t_{2}$ implies $G_{t_{1}} \neq G_{t_{2}}$.

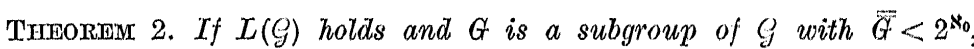
then there exists such an element $x \in \mathcal{G}$, which is of infinite order, and such that the naiural homomorphism $G *[(x)] \rightarrow[G \cup(x)]$ is an isomorphism $\left(^{5}\right)$.

Proof. Let us consider the class $\boldsymbol{M}$ of all subsets of $\mathcal{G}$ having one of the following forms

$$
\begin{aligned}
& \left\{x: x^{k}=1\right\}, \quad k=1,2, \ldots \\
& \left\{x: a_{1} x^{k_{1}} \ldots a_{m} x^{k_{m}}=1\right\} \quad\left(a_{i}, 7_{i} \text { and } m \text { as in }(1)\right) .
\end{aligned}
$$

It is clear that $\boldsymbol{M} \leqslant \ddot{G}+\kappa_{0}<2^{\aleph_{0}}$. Moreover, each set $A \in M$ is an analytic surface in $G$ (in the sense of [2]). In fact, for sets of the form (2) this is clear and for sets of the form (3) this follows from $L(Y)\left({ }^{0}\right)$. Therefore, by a theorem of [2], the set $\bigcup_{A \in M} A$ is a border set in $G$ (i.e. itis complement is dense in $\mathcal{Y}$ ). It follows that there exists an element $x \in \mathcal{Y} \bigcup_{A \in M} A$. Olearly, $x$ satisfies the conclusion of Theorem 2; q.e.d.

LEMma 1. If $L(\mathcal{G})$ holds and $G$ and $H$ are subgroups of $g$ with $\bar{G}, \widetilde{\vec{H}}<2^{s_{0}}$, then there exists an $x \in \mathcal{G}$ such that the natural homomorphism $G * H$ $\rightarrow\left[G \cup x H x^{-1}\right]$ is an isomorphism.

Proof. By Theorem 2 there exists an $x \in \mathcal{G}$ such that the natiual homomorphism $[G \cup H] *[(x)] \rightarrow[G \cup H \cup(x)]$ is an isomorphism. It is clear that for such an $x$ the conclusion of Lemma 1. holds; q.e.d.

TheOREM 3. If $L(Y), \tau \in T$, and $G_{t}(t \in T)$ is a system of subgroups of $g$ with $\overline{G_{t}}<2^{N_{0}}$ for any $t \in T$ and $\bar{T} \leqslant 2^{N_{0}}$, then there exists a system $\left(x_{t}\right)_{t \in T} C \mathcal{C}$ with $x_{\tau}=1$, such that the natural homomorphism $\prod_{t \in T^{\prime}}^{*} G_{t} \rightarrow\left[\bigcup_{t \in I^{\prime}} x_{t} G_{t} x_{t}^{-1}\right]$ is an isomorphism.

Proof. The set $T$ can be well ordered in a sequence $\left(t_{\xi}\right)_{\xi<x}$ such that $\tau=t_{0}$ and

$$
\sum_{\xi \leqslant \alpha} \overline{\bar{G}}_{t_{\xi}}<2^{N_{0}} \quad \text { for any } \quad \alpha<x\left({ }^{7}\right)
$$

Then by means of a simple induction on the basis of Lemma 1 . wo find a sequence $\left(x_{t_{\xi}}\right)_{\xi<x} \subset g$ for which the conelusion of Theorem 3 holds; (1.e.d.

(5) $A * B$ denotes the free product of the groups $A$ and $R$. This theorem waA conjectured ([17]) for (; locally compact connected and simple. Of courso such a $; ;$ is a Lie group (by the approximation theorem of II. Yamabo - see [14.], p. 17n). J'his problem remains open.

${ }^{\circ}$ Since the mapping $x \rightarrow a x k_{1} \ldots a_{m} x^{k_{m}}$ is analytic, which is woll knownsee e.g. [15], proof of Lemma 2.

(7) This follows from [16], Lemma 2 (a result of \$. Swierczkowski). Yt is interesting that this result replaces in some sense the regularity of the cardinal $2^{\aleph_{0}}$, which belongs of course to the classical conjectures of set theory.

Fundamenta Mathematicae, T. L (1961) 
Remark 1. In Lemma 1 and Theorem 3 , a supposition $L^{\prime}(G)$ weaker than $L(G)$ would be sufficient. $L^{\prime}(G)$ is obtained by replacing (1) in $L(\zeta)$ by

$$
a_{1} x b_{1} x^{-1} a_{2} x b_{2} x^{-1} \ldots a_{n} x b_{n} x^{-1} \quad \text { with } \quad a_{i}, b_{i} \in \mathcal{S}(1) \text { and } n \geqslant 1 .
$$

Problem. Does $L(\mathcal{G})$ or $L^{\prime}(\mathcal{G})$ hold true for every connected simple Lie group $\mathrm{g}$ ?

Proof of Theorem 1. The statements $L\left(O_{3}\right)$ and $L(\%)$ will be proved in Section 3; therefore Theorem 1. follows from Theorem 3.

Remark 2. One can obtain results analogous to Whoorem 2 and 3 for any connected locally compact group 9 supposing $L(Y)$ holds if one uses the continuum hypothesis or if one supposes the stronger inoqualities $\overline{\bar{G}} \leqslant \aleph_{0}, \overline{\bar{G}}_{t} \leqslant \aleph_{0}$ for any $t \in T$ and $\bar{T} \leqslant \aleph_{1}$. The proofs of such theorems are analogous: one has to use the theorem of Baire on the setis of the first category instead of the theorem of [2], and the only point to be completed is to prove that all sets of the form (2) or (3) are nowhere dense in $g$. But this clearly follows on account of $L(G)$ from [15], Theorem 1 , and the approximation theorem (1.c. $\left.\left({ }^{5}\right)\right)$.

3. The statement $L\left(\mathrm{O}_{3}\right)$ visibly follows from the following

LEMMA 2. If $k_{1}, \ldots, k_{n}$ are integers different from $0, a_{1}, \ldots, a_{n} \in \mathrm{O}_{3}$, and $x_{\varphi} \in O_{3}(0 \leqslant \varphi<2 \pi)$ where $\varphi$ is the rotation angle of $x_{\varphi}$ and all $x_{p}$ have a common rotation axis $L$ and $a_{s}(L) \neq I$ for $s=2, \ldots, n$ then the matrix

$$
a_{1} x_{\varphi}^{k_{1}} a_{2} x_{\varphi}^{k_{2}} \ldots a_{n} x_{p}^{k_{n}}
$$

is a non constant function of $\varphi$.

Proof. We can suppose from now on without loss of generality that $a_{1}=1$ and that we have chosen in $\chi^{3}$ a coordinate system, such that the third coordinate axis is the line $L$.

Now we need some auxiliary statements.

(A) The following conditions are equivalent (for a $\in \mathrm{O}_{3}$ )

(i) $a(L) \neq L$;

(ii) $L \neq($ axis of $a$ ) and, if the rotation angle of $a$ is $\pi$, then also $L$ is not perpendicular to the axis of $a$

(iii) $a=\left(a_{i j}\right)_{i, j=1,2,3}$ and

$\left(a_{11}-a_{22} \neq 0\right.$ or $\left.a_{12}+a_{21} \neq 0\right)$ and $\left(a_{11}+a_{22} \neq 0\right.$ or $\left.a_{12}-a_{21} \neq 0\right)$.

The equivalences (i) $\leftrightarrow$ (ii) and (ii) $\leftrightarrow$ (iii) are elementary. have

(B) For any linear transformation $a=\left(a_{i j}\right)_{i, j=1,2}$ of the plane $\Re^{2}$ we

$$
a(z)=A z+B \bar{z}\left({ }^{8}\right),
$$

(8) We identify the point $(\xi, \eta) \in \Re^{2}$ with the complex number $z=\xi+i \eta$, and the point $(\xi,-\eta) \epsilon \mathcal{R}^{2}$ with $\bar{z}=\xi-i \eta$. where

and

$$
\begin{aligned}
& A=\frac{1}{2}\left[\left(a_{11}+a_{22}\right)+i\left(a_{21}-a_{12}\right)\right], \\
& B=\frac{1}{2}\left[\left(a_{11}-a_{22}\right)+i\left(a_{12}+a_{21}\right)\right], \\
& a_{11}= \operatorname{Re}(A+B), \quad a_{12}=-\operatorname{Im}(A-B), \\
& a_{21}=\operatorname{Im}(A+B), \quad a_{22}=\operatorname{Re}(A-B) .
\end{aligned}
$$

This is elementary.

(C) All the functions $\cos ^{n} p$ and $\sin p \cos ^{n} p(n=0,1,2, \ldots)$ are independent, i.e. a finite linear form formed of them vanishes if and only if all the coefficients vanish.

This follows by independence of $\cos ^{n} \varphi(n=0,1,2, \ldots)$ and the fact that $\cos ^{n} \varphi$ are even functions and $\sin \varphi \cos ^{n} \varphi$ are odd functions.

(D) $x_{p}^{k}=2^{|k|-1} \cos ^{|k|-1} \varphi\left(\begin{array}{ccc}\cos \varphi & -\sigma \sin \varphi & 0 \\ \sigma \sin \varphi & \cos \varphi & 0 \\ 0 & 0 & 0\end{array}\right)+b, \quad(k= \pm 1, \pm 2, \ldots)$,

where $\sigma=\operatorname{sgn} k$ and $b$ is a matrix with elements of the form

$$
\sum_{r=0}^{|k|-2} \alpha_{r} \sin \varphi \cos ^{r} \varphi+\sum_{r=0}^{|k|-1} \beta_{r} \cos \varphi^{r}
$$

This follows from the known formulae

$$
\begin{aligned}
& \cos n \varphi=2^{n-1} \cos ^{n} \varphi+P_{1}, \\
& \sin n \varphi=\sin \varphi\left(2^{n-1} \cos ^{n-1} \varphi+P_{2}\right),
\end{aligned}
$$

(where $P_{1}$ resp. $P_{2}$ are polynomials in $\cos \varphi$ of degree $<n$ resp. $n-1$ ) and from the equality

$$
x_{\varphi}^{k}=\left(\begin{array}{ccc}
\cos k \varphi & -\sin k \varphi & 0 \\
\sin k \varphi & \cos k \varphi & 0 \\
0 & 0 & 1
\end{array}\right) .
$$

For any matrix $a=\left(a_{i j}\right)_{i, j=1,2,3}$ we take the notation

$$
a^{*}=\left(a_{i j}\right)_{i, j=1,2} \text {. }
$$

(E) (i) The elements of the matrix (4) can be represented uniquely in the form

$$
\sum_{r=0}^{d-1} \alpha_{r}^{i j} \sin \varphi \cos ^{r} \varphi+\sum_{r=0}^{d} \beta_{r}^{i j} \cos ^{r} \phi \quad(i, j=1,2,3)
$$

where $d=\left|k_{1}\right|+\ldots+\left|k_{n}\right|$.

(ii) The elements of the matrix

$$
c=\left(c_{i j}\right)_{i, j=1,2}=\prod_{s=1}^{n} a_{s}^{*}\left(\begin{array}{cc}
\cos \varphi & -\sigma_{s} \sin \varphi \\
\sigma_{s} \sin \varphi & \cos \varphi
\end{array}\right)
$$


where $\sigma_{s}=\operatorname{sgn} k_{s}$, can be represented uniquely in the form

$$
c_{i j}=\sum_{r=0}^{n-1} \gamma_{r}^{i j} \sin \varphi \cos ^{r} \varphi+\sum_{r=0}^{n} \delta_{r}^{i j} \cos ^{r} \varphi \quad(i, j=1,2) .
$$

(iii) $\quad \alpha_{d-1}^{i j}=2^{d-n} \gamma_{n-1}^{i j}$ and $\beta_{d}^{i j}=2^{d-n} \delta_{n}^{i j}(i, j=1,2)$.

The statements (i) and (ii) can be easily proved by induction on $n$ if one applies (C), the formulae (5) and $\sin ^{2} \varphi=1-\cos ^{2} \phi$. The statement (iii) follows in the same way from (O) and (D).

Now, by (O) and (E) (i), for proving Lomma 2 it is enough to show that one of the terms $\alpha_{d-1}^{i j} \sin p \cos ^{d-1} p+\beta_{d}^{i j} \cos ^{d} p(i, j=1,2,3)$ does not vanish. By (E) (iii) it is enough to prove that one of tho terms $\gamma_{n-1}^{i j} \sin \varphi \cos ^{n-1} \varphi+\delta_{n}^{i j} \cos ^{n} \varphi(i, j=1,2)$ does not vanish.

We have

and we put

$$
a_{1}^{*}(z)=z, \quad x_{\varphi}^{*}(z)=e^{\varphi} z,
$$

$$
\begin{aligned}
a_{s}^{*}(z) & =A_{s} z+B_{s} \bar{z} \quad(s=2,3, \ldots, n), \\
c(z) & =C z+D \bar{z} .
\end{aligned}
$$

Then by (A), the supposition $a_{s}(L) \neq L$ of the Lemma 2 and (B) (i) we have

(6)

$$
A_{8} \neq 0 \neq B_{8} \quad(s=2,3, \ldots, n) .
$$

By the definition ((E) (ii)) of the matrix 0 wo have

$$
\begin{aligned}
& C=\sum O_{\varkappa_{2}, \ldots, \varkappa_{n}} e^{i\left(\sigma_{2}+\varkappa_{2} \sigma_{2}+\ldots+\varkappa_{n} \sigma_{n}\right) \varphi}, \\
& D=\sum D_{\varkappa_{2}, \ldots, \varkappa_{n}} e^{i\left(\sigma_{1}+\varkappa_{2} \sigma_{2}+\ldots+\varkappa_{n} \sigma_{n}\right) \varphi},
\end{aligned}
$$

where the sums are running over all sequences $x_{2}, \ldots, x_{n}$ with $x_{s}=-1$ and $C_{x_{2}, \ldots, x_{n}}$ and $D_{x_{2}, \ldots, x_{n}}$ are products of some of the number's $A_{8}$ and $B_{s}$ and their conjugates. Clearly, by $(6)^{*}$ all the numbers $O_{x_{0}} A_{8}$ and $D_{x_{2}, \ldots, x_{n}}$ are different from 0 . In each of the sums (7) there is oxactly one term in which $\sigma_{1}+x_{2} \sigma_{2}+\ldots+x_{n} \sigma_{n}=\sigma_{1} n$ (since this holds if and only if $\varkappa_{s}=\sigma_{1} \sigma_{s}$ ). Hence $O$ and $D$ contain one term $K e^{i \sigma_{1} n \varphi}$ and $L e^{i \sigma_{1} n \varphi}$ respectively with $K \neq 0 \neq L$. Therefore by (B) (ii) we have

$$
\begin{array}{ll}
c_{11}=\operatorname{Re}\left[e^{i \sigma_{1} n \varphi}(K+L)+t_{11}\right], & c_{12}=-\operatorname{Im}\left[e^{i \sigma_{1} n \varphi}(K-L)+t_{12}\right], \\
c_{21}=\operatorname{Im}\left[e^{i \sigma_{1} n \varphi}(K+L)+t_{21}\right], & c_{22}=\operatorname{Re}\left[e^{i \sigma_{1} n \varphi}(K-L)+t_{22}\right],
\end{array}
$$

where $t_{i j}$ are trigonometrical sums of degree $<n$.

Then one of the $c_{i j}$, when represented in the form (I) (ii), contains a non-vanishing term $\gamma_{n-1}^{i f} \sin \varphi \cos ^{n-1} \varphi+\delta_{n}^{i j} \cos ^{n} \varphi$, which concludes the
proof.
LEMEMA 3. $L(m)$ holds.

Proof. The mapping

$$
\left(\begin{array}{ll}
a & b \\
c & d
\end{array}\right) \rightarrow \frac{a z+b}{c z+d}
$$

is a homomorphism of the unimodular group of matrices onto $M$ corresponding to the identification of matrices of opposite sign. Keeping in mind this fact we can work with matrices.

We shall prove that the matrix (all matrices considered are unimodular)

$$
\prod_{s=1}^{n} a_{s} x^{k_{s}}, \quad \text { where } \quad a_{s}=\left(\begin{array}{ll}
\alpha_{s} & \beta_{s} \\
\gamma_{s} & \delta_{s}
\end{array}\right) \neq \pm\left(\begin{array}{ll}
1 & 0 \\
0 & 1
\end{array}\right) \quad(s=1, \ldots, n)
$$

( $k_{s}$ are integers different from $0, n \geqslant 1$ ), depends on the matrix $x$.

We can suppose without loss of generality that $\gamma_{s} \neq 0$ for $s=1, \ldots, n$ (using an inner automorphism of the group). Let us verify under this assumption that the matrix

$$
\left(\pi_{i j}(t)\right)_{i, j=1,2}=\prod_{s=1}^{n} a_{s} x_{t}^{k_{s}}, \quad \text { where } \quad x_{t}=\left(\begin{array}{ll}
1 & t \\
0 & 1
\end{array}\right),
$$

depends on $t$. Clearly

$$
a_{s} x_{t}^{k_{s}}=\left(\begin{array}{ll}
\alpha_{s} & \alpha_{s} k_{s} t+\beta_{s} \\
\gamma_{s} & \gamma_{s} k_{s} t+\delta_{s}
\end{array}\right)
$$

By an easy induction on $n$ we verify that

$$
\pi_{22}(t)=\gamma_{1} \ldots \gamma_{n} k_{1} \ldots k_{n} t^{n}+P,
$$

where $P$ is a polynomial in $t$ of degree $<n$; q.e.d.

4. In this section we give some more special results on $\mathcal{O}_{3}$ which are easy consequences of Lemma 2.

THEOREM 4. Let $G$ be a subgroup of $\mathcal{O}_{3}$ and $\varphi$ a fixed angle with $0<\varphi<2 \pi$ and $\varphi \neq \pi$, and $Q$ an axis (a plane) in $\mathcal{R}^{3}$ containing the origin o and such that $a(Q) \neq Q$ for every $a \in G \backslash(1)$. Then every rotation $r \in O_{3}$ with axis $Q$ (with axis contained in $Q$ and rotation angle $\varphi$ ), except a set of $\bar{G}+s_{0}$ such rotations, is such that the natural homomorphism $G *[(r)] \rightarrow[G \cup(r)]$ is an isomorphism.

Proof. The first case clearly follows from the Lemma 2 since every function

$$
a_{1} r k_{1} a_{2} r^{k_{2}} \ldots a_{n} r^{k_{n}}
$$

$\left(k_{i}\right.$ integers different from 0 and $a_{i} \in G \backslash(1), n \geqslant 1$ ) is analytical and depends on the rotation angle of $r$ the axis being $Q$. 
In the second case we consider the product

$$
a_{1} x b^{k_{1}} x^{-1} a_{2} x b^{k_{2}} x^{-1} \ldots a_{n} x b^{k_{n}} x^{-1}
$$

where $b$ is a fixed rotation with axis contained in $Q$ and angle $p$, and $x$ is a rotation with axis perpendicular to $Q$ and variable angle. Putting $r=x b x^{-1}$ we obtain rotations with axis in $Q$ and angle $p$. Then we obtain the conclusion since, by Lemma $2,(8)$ depends essentially and analytically on the rotation angle of $x$.

Remark 3. Theorem 4 is a refinement of Theorem 2 tor $G=\mathrm{O}_{3}$.

THEOREM 5. If $x_{p}$ and $y_{p}$ are rotations with fixed different axes and variable common rotation angle $p$, then with the exooption of an at most denumerable set of $p-s x_{p}$ and $y_{p}$ are free generators of a free group $\left({ }^{9}\right)$.

Proof. It is visible that there exist an $a \in O_{3}$, such that $y_{p}=a x_{p} a^{-1}$ for all $\varphi$-s. Therefore, by Lemma 2, the function

$$
x_{\varphi}^{k_{1}} y_{\varphi}^{l_{1}} x_{\varphi}^{k_{2}} y_{\varphi}^{l_{2}} \ldots x_{\varphi}^{k_{n}} y_{\varphi}^{l_{n}}=x_{\varphi}^{k_{1}} a x_{\varphi}^{l_{1}} a^{-1} x_{\varphi}^{\gamma_{l_{2}}} a x_{\varphi}^{l_{2}} a^{-1} \ldots x_{\varphi}^{k_{n}} a x_{\varphi}^{l_{n}} a^{-1}
$$

depend essentially and analytically on $p$, and the Theorem 5 follows.

\section{, References}

[1] S. Balcerzyk and Jan Myoielski, Some theorems on the representations of free products, Bull. Acad. Polon. Sci. Cl. IIr. 5 (1957), p. 1029-1030.

[2] - - On the method of category in analytic manifolds, Fund. Math. 44 (1957), pp. $295-299$.

[3] - On the existence of free subgroups in topological groups, ibidem,

[4] N. G. de Bruijn, A theorem on choice functions, Indagationes Math. 19 (1957), pp. $409-411$.

[5] - Embedding theorems for infinite groups, ibidem, p. 560-569.

[6] - Addendum to "A theorem on choice functions", ibidem, 21 (1959), p. 327.

[7] Th. J. Deklzer, On reflections in Euclidean spaces generating free products, Nieuw Archief voor Wiskunde 7 (1959), pp. 57-60.

[8] - Paradoxical decompositions of sets and spaces, Thesis, Amsterdam 1958. pp. $67-69$.

(1959)

[10] K. Golberg and M. Newman, Pairs of matrioes of oriler two whioh generate free groups, Illinois J. of Math. 1 (1957), pp. 44644.48.

[11] J. de Groot, Orthogonal isomorphio representations of free groups, Canadian $56 \cdot 262$.

[12] F. Hausdorff, Grundzüge der Mengenlehre, Liepzig 1914, pp. 4:69-472. 113] K. A. Hirsch, Appendix B. A. G. Kurosh, The theory of groups, New York,
1956, pp. 261-264.

(9) This was conjectured by J. de Groot ([11]). Th. J. Dekker has shown ([9]) more than this: $x_{\varphi}$ and $y_{\varphi}$ are free generators if $\cos \varphi$ is transcendental.
[14] D. Montgomery and L. Zippin, Topological transformation groups, New York 1955.

[15] Jan Mycielski, On the extension of equalities in connected topological groups, Fund. Math. 44 (1957), pp. 300-302.

[16] - About sets invariant with respect to denumerable changes, ibidem, 45 (1958), pp. $295-305$

[17] - Problem 206, Coll. Math. 5 (1957), p. 119.

[18] - and $\mathrm{s}$. Euclidean space, Fund. Math. 45 (1958), pp. 283-291.

[19] J. von Neumann, Zur allgemeinen Theorie der Masses, ibidem, 13 (1929), pp. $73-116$.

[20] W. Sierpiński, Sur le paradoxe de la sphère, ibidem, 33 (1945), pp. 235-236.

Regu par la Rédaction le 18. 8.1960 